Document downloaded from:

http://hdl.handle.net/10251/159835

This paper must be cited as:

Marco, M.; Infante-Garcia, D.; Diaz-Alvarez, J.; Giner Maravilla, E. (2019). Relevant factors affecting the direction of crack propagation in complete contact problems under fretting fatigue. Tribology International. 131:343-352. https://doi.org/10.1016/j.triboint.2018.10.048

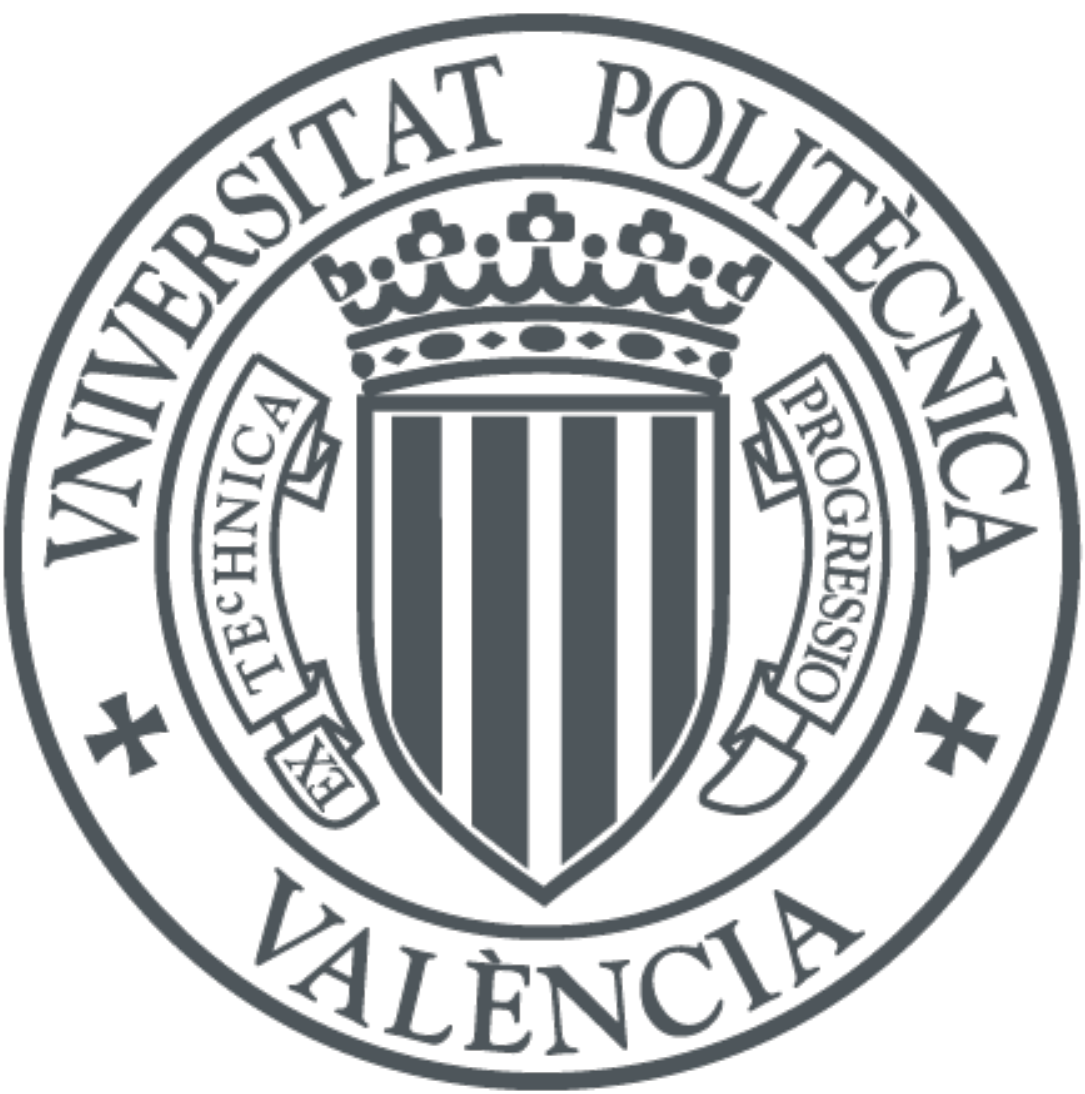

The final publication is available at

https://doi.org/10.1016/j.triboint.2018.10.048

Copyright Elsevier

Additional Information 


\title{
Relevant factors affecting the direction of crack propagation in complete contact problems under fretting fatigue
}

\author{
Miguel Marco ${ }^{\mathrm{a}}$, Diego Infante-García ${ }^{\mathrm{a}}$, José Díaz-Álvarez ${ }^{\mathrm{a}}$, \\ Eugenio Giner ${ }^{\mathrm{b}, *}$ \\ ${ }^{a}$ Depto. de Ingeniería Mecánica, Universidad Carlos III de Madrid, Avda. de la \\ Universidad 30, 28911-Leganés, Madrid, Spain. \\ ${ }^{b}$ Centro de Investigación en Ingeniería Mecánica - CIIM, \\ Depto. de Ingeniería Mecánica y de Materiales, \\ Universitat Politècnica de València, Camino de Vera, 46022 Valencia, Spain.
}

\begin{abstract}
In fatigue problems, an accurate estimation of the propagation direction is important for life prediction. We identify the most relevant factors that affect the crack orientation during the propagation stage of fretting fatigue cracks, arising from complete contacts. Contrary to what initially expected, parameters such as normal load, cyclic bulk load, etc. do not have a noticeable influence on the orientation. However the relative Young's moduli of indenter/specimen materials, the indenter width and the surface coefficient of friction are the most influencing factors. Analyses are performed through the extended finite element method (X-FEM) and an orientation criterion for non-proportional loading proposed by the authors. Experimental fretting fatigue tests confirm the predicted trends. An explanation of this behaviour is also given.

Keywords: Fretting fatigue; complete contact; crack propagation;

orientation criterion; extended finite elements.
\end{abstract}

\footnotetext{
${ }^{*}$ Corresponding author. Tel.: +34-96-3877007 ext. 76218; fax: +34-96-3877629.

Email address: eginerm@mcm.upv.es (Eugenio Giner)

Preprint submitted to Tribolology Int

October 8, 2018
} 


\section{1. Introduction}

$6 \quad$ Fretting problems are found in many mechanical components and are 7 often responsible for accelerating an eventual fatigue failure. They are 8 characterized by the existence of two or more solids in contact that un9 dergo relative displacements and they can be broadly classified into fretting 10 fatigue and fretting wear problems. One of the main features of fretting 11 fatigue problems is that cracks initiate next to the contact zone typically 12 under conditions of partial slip [1]. After the initiation stage, cracks usually 13 propagate under the cyclic stress field existing relatively far from the con14 tact region. On the other hand, fretting wear situations often hinder crack 15 propagation, as the incipient nucleated cracks are erased by material wear due to gross slip conditions [2] and there is no far field cyclic stress that 17 promotes crack growth. This paper focuses on the study of complete contact problems under fretting fatigue in metals, and more specifically, on the 19 crack propagation stage, i.e. the stage when the crack is already nucleated and its length is several times greater than the typical grain size.

Compared to the plain fatigue endurance for the same materials, fretting 22 fatigue lives are substantially reduced. The main reason is that the contact 27 life.

When the propagation life of a fretting crack is to be estimated, numerical methods such as the extended finite element method (X-FEM) [5-8] or standard FEM with remeshing techniques [9] can be used to model the 
31 crack presence under the combined influence of the bulk load and contact

32 stresses. Then, fracture mechanics approaches and crack growth laws can

33 be applied to estimate the remaining life, e.g. $[7,10,11]$ in combination

34 with methods that predict the initiation life, such as the variable initiation

35 length approach [12]. Recently, the prediction of crack location and orien-

36 tation in the initiation stage has been addressed in [13] using a cohesive

37 zone model approach (CZM) in conjunction with XFEM. Another proposal

38 to predict the crack initiation direction is given in [14], where a method is

39 developed by calculating the average values of the normal and shear stresses

40 along a critical prospective direction.

${ }_{41}$ In this work, a complete contact fretting fatigue configuration as the 42 one sketched in Fig. 1 is considered. In a complete contact, the contacting 43 area is independent of the normal load $P$ due to the abrupt change of the 44 indenter geometry, as opposed to incomplete contacts, such as Hertzian 45 contacts. Some of the parameters affecting the loading conditions are the 46 normal load $P$, the tangential load $Q$, the cyclic bulk load $\sigma_{\text {Bulk }}$ and its ${ }_{47}$ corresponding stress ratio $R$ (defined as $R=\sigma_{\text {Bulk,min }} / \sigma_{\text {Bulk,max }}$ ) and the 48 friction coefficient between the contacting solids.

49 A question arises regarding the influence of the different fretting param50 eters on the crack orientation. No information was found in the literature 51 regarding this issue. This work makes use of the numerical tools such as $52 \mathrm{X}-\mathrm{FEM}$ and the orientation criterion for non-proportional loading condi53 tions proposed in [15] to study the relevant parameters that affect crack 54 orientation. The X-FEM enables the parametric study of different fret55 ting configurations (loading, materials, etc.) in a straightforward way, as 56 the crack growth is simulated automatically without the need of remeshing 


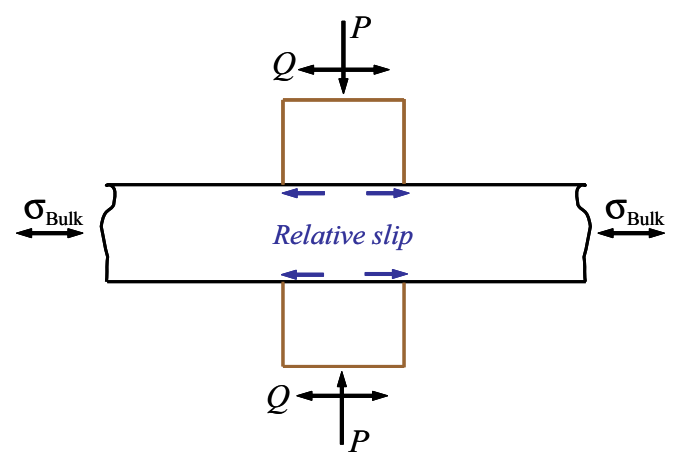

Figure 1: Sketch of the main loads acting in a complete contact fretting fatigue problem, as the one analyzed in this work.

$57[16,17]$.

${ }_{58}$ As shown in experimental tests carried out by the authors [15] (see 59 Fig. 2) and in many works in the literature, e.g. [18, 19], cracks emanating

60 from the edge of a contact pressing onto a surface tend to grow with a

${ }_{61}$ slight deviation inwards beneath the contact and not fully perpendicular

62 to the applied bulk stress. This slight deviation from the normal direction

63 cannot be predicted using a conventional orientation criterion, such as the

64 maximum tangential or hoop stress criterion (MTS) and this is the main

65 motivation of this research.

${ }_{66}$ The objective of the work is to identify the relevant parameters affecting

${ }_{67}$ the crack path orientation. A parametric study of some a priori relevant

68 magnitudes is carried out, such as normal load on the indenters, bulk load

69 on the specimen, stress ratio, relative stiffness of the indenter and specimen

70 materials, coefficient of friction and indenter width. This is performed by

71 benefiting from the main advantage of XFEM, i.e. crack remeshing is not

72 necessary for simulating crack propagation. Contrary to previous expecta-

73 tions, it is shown that the relative magnitude of the applied loads has no 

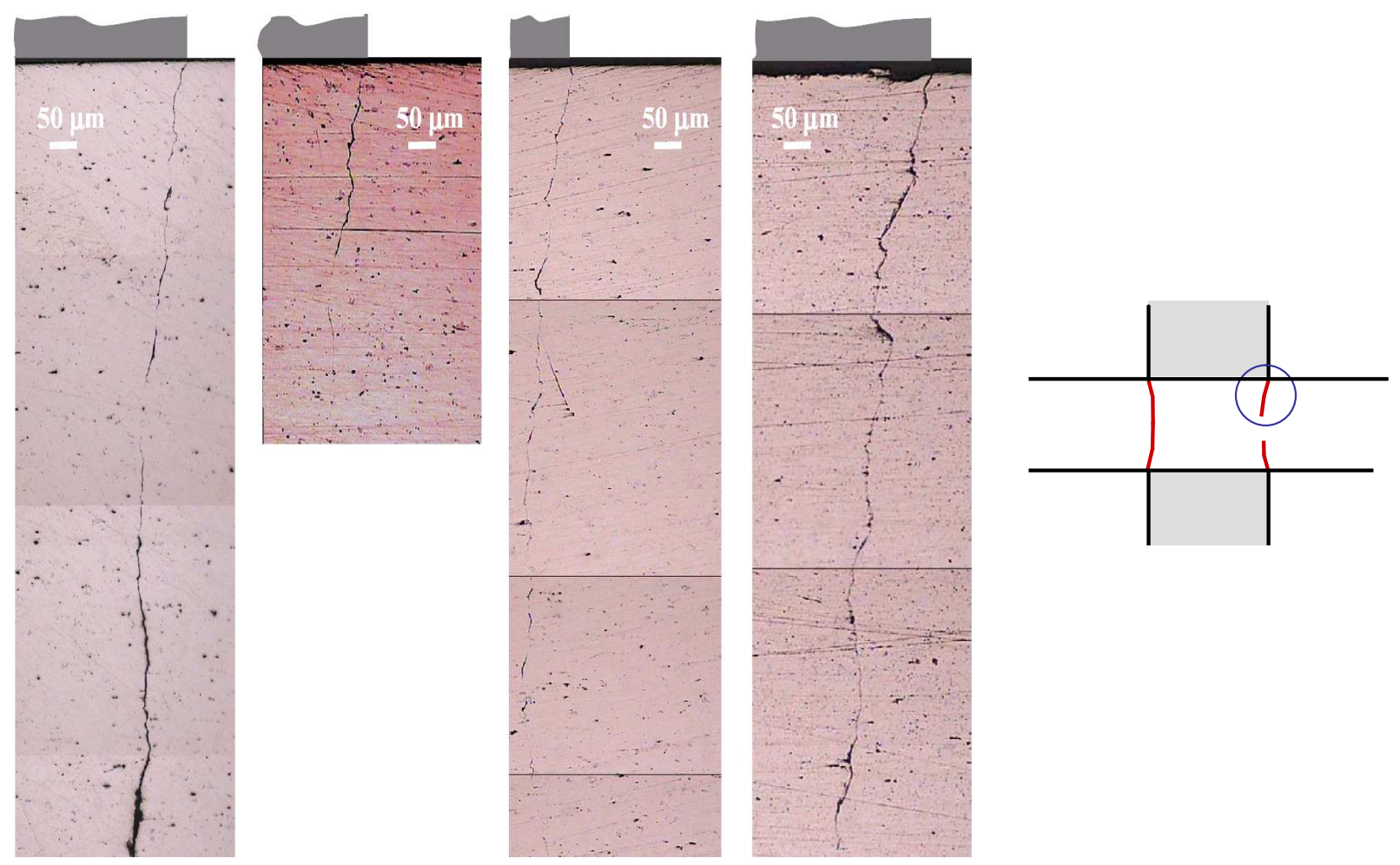

Figure 2: Propagation of non-failure cracks of four tests [15], emanating from the edge of contact. The material of both indenter and specimen is Al 7075-T6. Loads of each test: from left to right, $\sigma_{P}=40,80,80,160 \mathrm{MPa}$ and $\sigma_{\text {Bulk,max }}=110,130,150,190$ MPa with $R=-1$, load-controlled. Frequency of the fatigue tests is $15 \mathrm{~Hz}$.

74 significant effect on crack orientation although, of course, it does on the

75 fatigue life. However, it is found that the indenter width, the friction coef-

76 ficient between the indenter and the specimen and the stiffness ratio of the

77 indenter with respect to the specimen have a noticeable effect.

\section{2 . The criterion of the minimum shear stress range}

79 In order to predict correctly the path followed by the crack, it is impor-

80 tant to apply an orientation criterion that considers the nonproportional 81 evolution of loads in fretting fatigue problems. Usually, the contact loads 
82 do not evolve proportionally to the cyclic bulk loads. This means that 83 the principal stress directions and the stress intensity factor ratio $K_{\mathrm{II}} / K_{\mathrm{I}}$ 84 change with time, invalidating the application of the classical MTS crite85 rion [20]. An orientation criterion for nonproportional loading conditions was proposed by the authors in [15], where a brief review of other criteria

87 for nonproportional loading is also included. It was shown that the correct 88 application of a criterion of this kind is essential to agree with experimental 89 observations and a comparison is provided with the MTS criterion. Indeed, 90 the MTS criterion was applied in one of our first works [5], leading to wrong 91 estimations of the crack path. This was also found in [21].

\subsection{Fundamentals of minimum shear stress range criterion}

For the geometric and loading configuration considered in this work, the crack remains closed during a large part of the loading cycle, as verified from the numerical analyses. Assuming an elastic behaviour, the stress state under crack face contact conditions must be essentially controlled by $K_{\mathrm{II}}$, the only stress intensity factor that can exist for a totally closed crack in 2D. The criterion applied in this work [15] is a generalization for nonproportional evolutions of the so-called criterion of local symmetry, well established for proportional loading by Goldstein and Salganik [22] and Cotterell and Rice [23]. The criterion of local symmetry states that the crack will propagate in the direction where $K_{\mathrm{II}}=0$. For nonproportional loading, the condition $K_{\text {II }}=0$ cannot be reached on the same plane along the whole cycle, and therefore, the proposed criterion seeks the angle for which the range $K_{\text {II }}$ is minimized along the cycle. This hypothesis obviously reduces to the condition $K_{\mathrm{II}}=0$ when applied to proportional loading problems. It is worth 
noting that, for proportional loading, $K_{\mathrm{II}}=0$, Nuismer and MTS criteria lead to very similar results $[23,24]$. Sumi $[25,26]$ gives an interesting comparison of the results provided by different orientation criteria. In practice, computing $K_{\text {II }}$ values under crack face contact must include the effect of friction tractions on crack faces, as in [27, 28], which can be cumbersome and prone to inaccuracies when using domain and contour integrals. Instead, and equivalently for the application of the minimum shear stress range criterion, we search for the angle that minimizes the shear stress range at the crack tip, $\min (\Delta \tau)$. Shear stresses develop always in two orthogonal planes and there are two orthogonal planes on which the range is minimum, $\min (\Delta \tau)$. From these two possible crack growth directions, we choose the plane with the maximum $\Delta \sigma_{\mathrm{n}}$, because it is the plane where less frictional energy is lost and there is more energy available for crack propagation. This approach is in line with the principle that a crack will grow in the direction which maximizes the strain energy release rate $G[23,24]$.

As verified in [15] and also in this work, the $\min (\Delta \tau)$ direction coincides with the direction of the maximum range of normal stress, $\max \left(\Delta \sigma_{\mathrm{n}}\right)$. This is due to the in-plane stress tensor transformation that yields both extremes in the same direction, although this may not be the general case. However, one should notice that the criterion based on $\max \left(\Delta \sigma_{\mathrm{n}}\right)$ is inconsistent, since compressive stresses (usually present during a large portion of the fretting load cycle) do not contribute to crack propagation. Moreover, the direction predicted by the maximum range of the effective normal stress, $\max \left(\Delta \sigma_{\mathrm{n} \text {,eff }}\right)$, i.e. considering only the positive part of $\sigma_{\mathrm{n}}$, does not lead to good results at least in the problems studied by the authors, despite the intuitive idea that only the positive normal stresses will govern the crack 
propagation under an elastic material behaviour.

Fig. 3 sketches the convention used in the procedure. For each crack growth increment, the criterion is applied ahead the current crack tip and the prospective local direction is searched for which $\Delta \tau$ is minimum (see example of the estimation of the third increment direction in Fig. 3). In the results provided in this work, the predicted angle is reported with respect to a fixed reference: the angle is measured from the specimen surface. This way, a crack segment growing inwards (with respect to the indenter contact zone) has an angle $0^{\circ}<\theta<90^{\circ}$ and $-90^{\circ}<\theta<0^{\circ}$ indicates a crack segment growing outwards.
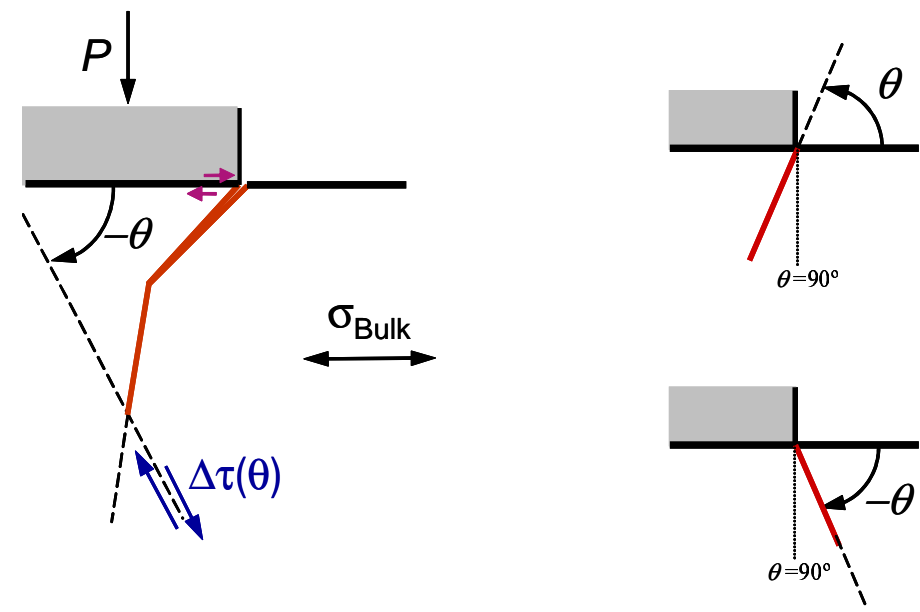

Figure 3: Application of the $\min (\Delta \tau)$ criterion to predict e.g. the third crack-growth increment direction. Sign convention for direction angles of a crack growth increment.

2.2. Application to a crack in a plate subjected to a tension-compression cycle

As an example of simple application of the minimum shear stress range criterion, a cracked bar of uniform section loaded in tension is analyzed. 
Our intention with this simple configuration is to illustrate that the criterion reduces to the expected angle of $90^{\circ}$ for such a simple case under proportional loading. Fig. 4 shows the geometry and loads of the model and a contour plot of the von Mises stress field. This preliminary analysis is performed using standard FEM with ABAQUS (no X-FEM is considered at this stage). Plane strain bilinear elements with four nodes and full integration are used (CPE4 in Abaqus). Inertial effects are not considered in this work and the fatigue crack propagation problem can be regarded as quasi-static. The material is modeled as linear elastic. The bulk load is cyclic with $R=-1$ and no indenter load exists in this simple example. The time evolution of the bulk load is similar to the one shown in Fig. 7 and it is also divided into four load steps.

Fig. 5, left, shows the variation of the normal stress $\sigma_{\mathrm{n}}$ and shear stress $\tau$ on a plane forming an angle $\theta$ with respect to the horizontal surface. Stresses are evaluated at finite elements located ahead the crack tip and transformed according to the angle of the prospective plane. The successive curves show the variation along time for the last load step (step 4 , i.e. between $t=3.0$ and $t=4.0)$. Note that the normal stress is maximum at the end of the step (curves located at the top of Fig. 5, left). This tensile stress is higher than the corresponding compressive stress due to the effect of the crack opening (mode I of fracture), whereas the closing stage does not concentrate such high stresses. The evolution of the shear stresses is analogous. Note that the shear stresses $\tau$ are zero for the angles $\theta$ where the normal stress is maximum or minimum (for any given increment of time). The fact that all the maxima and minima are attained at the same angles $\left( \pm 90^{\circ}\right.$ for $\sigma$ and $\pm 45^{\circ}$ for $\tau$ ) is indicative that the stresses evolve in a proportional way. If 

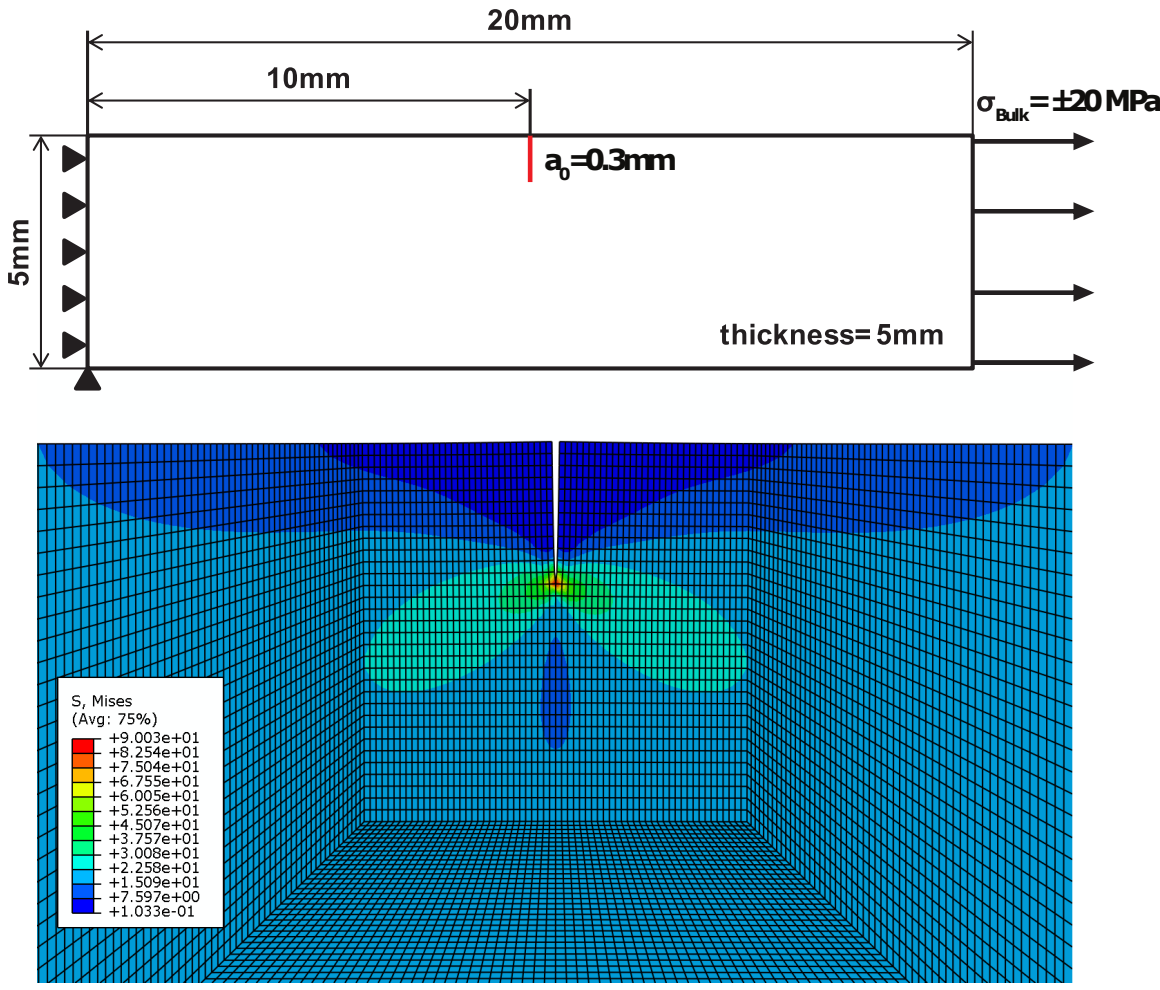

Figure 4: Top, geometry and loads of the model of a cracked bar in tension. Bottom, detailed view of a von Mises contour plot.

173 the time series of maxima and minima are shifted along the $\theta$ axis, then 174 the loads are nonproportional. Fig. 5 right, shows the application of the $175 \min (\Delta \tau)$ criterion. The same $\tau$ curves of Fig. 5, left, are replotted and the 176 maximum and minimum with time are marked in black. Then, the range of 177 variation $\Delta \tau$ is computed simply as $\Delta \tau=\tau_{\max }-\tau_{\min }$. The minimum shear stress range criterion predicts that the prospective propagation angles are 179 either $0^{\circ}$ or $90^{\circ}$ (there are always two prospective angles with a difference of $90^{\circ}$ ). The discrimination between both angles is done by choosing the 181 angle that also leads to the maximum normal stress to that plane. The 182 predicted angle of propagation for this case is $90^{\circ}$, as expected in such a 


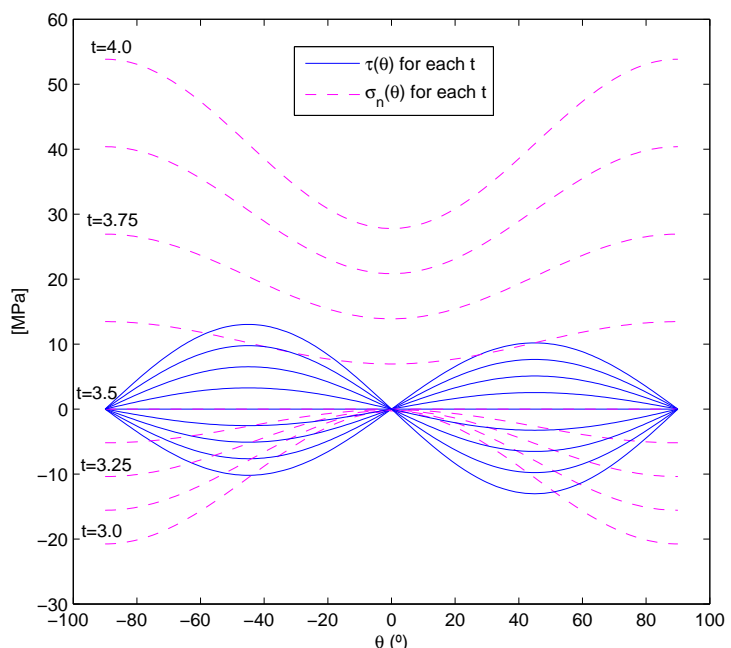

simple problem.

One advantage of the $\min (\Delta \tau)$ criterion over simply choosing the direction of $\max \left(\Delta \sigma_{\mathrm{n}}\right)$ is that the angle is detected sharply, as shown in Fig. 5. This sharpness of $\min (\Delta \tau)$ is also what governs the mechanics of the problem, leading to well defined propagation angles.

Figure 5: Left, variation of the normal stress $\sigma_{\mathrm{n}}$ and shear stress $\tau$ on planes forming a varying angle $\theta$ with respect to the horizontal surface. Stresses are evaluated at elements located ahead the crack tip. Right, application of the $\min (\Delta \tau)$ criterion.

\section{Numerical model}

Due to symmetry conditions, a quarter 2D finite element model has been considered to represent the fretting fatigue tests, as shown in Fig. 6. The rectangle $L \times b$ corresponds to the portion of the analyzed specimen and has a length of $L=4 b=20 \mathrm{~mm}$, the half width of the indenter $c$ is $5 \mathrm{~mm}$, and the distance between the contact plane and the point of the indenter at which loads are applied is $h=10 \mathrm{~mm}$. Four node, plane strain quadrilateral elements with full integration were used with a thickness $t=5 \mathrm{~mm}$. The 
smallest element size considered is $5 \mu \mathrm{m}$ at the right end of the contact zone.

The friction model assumed for the contact zone is a Coulomb model and the ABAQUS contact formulation based on Lagrange multipliers is used to model the contact between the indenter and the specimen. Unless otherwise stated, the friction coefficient between crack faces $\left(\mu_{\mathrm{CF}}\right)$ and between indenter and specimen $\left(\mu_{\mathrm{IS}}\right)$ is taken as $\mu_{\mathrm{CF}}=\mu_{\mathrm{IS}}=0.8$ [19]. The material behaviour is assumed linear elastic, despite the high stress concentration at the contact edge. The specimen material is an aluminium alloy $7075-$ T6, with $E=72 \mathrm{GPa}$ and $\nu=0.3$. For some of the cases analyzed, the indenter material is changed, as explained in Section 5.5. The application of the linear regime is deemed valid, due to the very small edge radius of the indenter and the relative high yield stress of the aluminium alloy. In addition, the loads simulated in this work are typical of high cycle fatigue, and therefore are nominally small (in contrast to high loads typical of low cycle fatigue problems). Therefore, the extent of the plastic zone at crack tip during crack propagation is relatively small and there is no noticeable plastic wake along crack faces. This is confirmed by the observation of the tested specimens, which showed no macroscopic evidence of plasticity (see micrographs in Fig. 2 in which crack faces match very well each other and also a view of the specimen contact surface in Fig. 7 of our previous work [10]). As a consequence, the existing plasticity is very localized and a small scale yielding assumption can be applied, analogous to the small scale yielding assumption admitted in linear elastic fracture mechanics (LEFM) around the crack tip.

The loading is considered quasistatic and its sequence is represented in Fig. 7 for one of the examples analyzed (case 3 of Table 1), where four load 


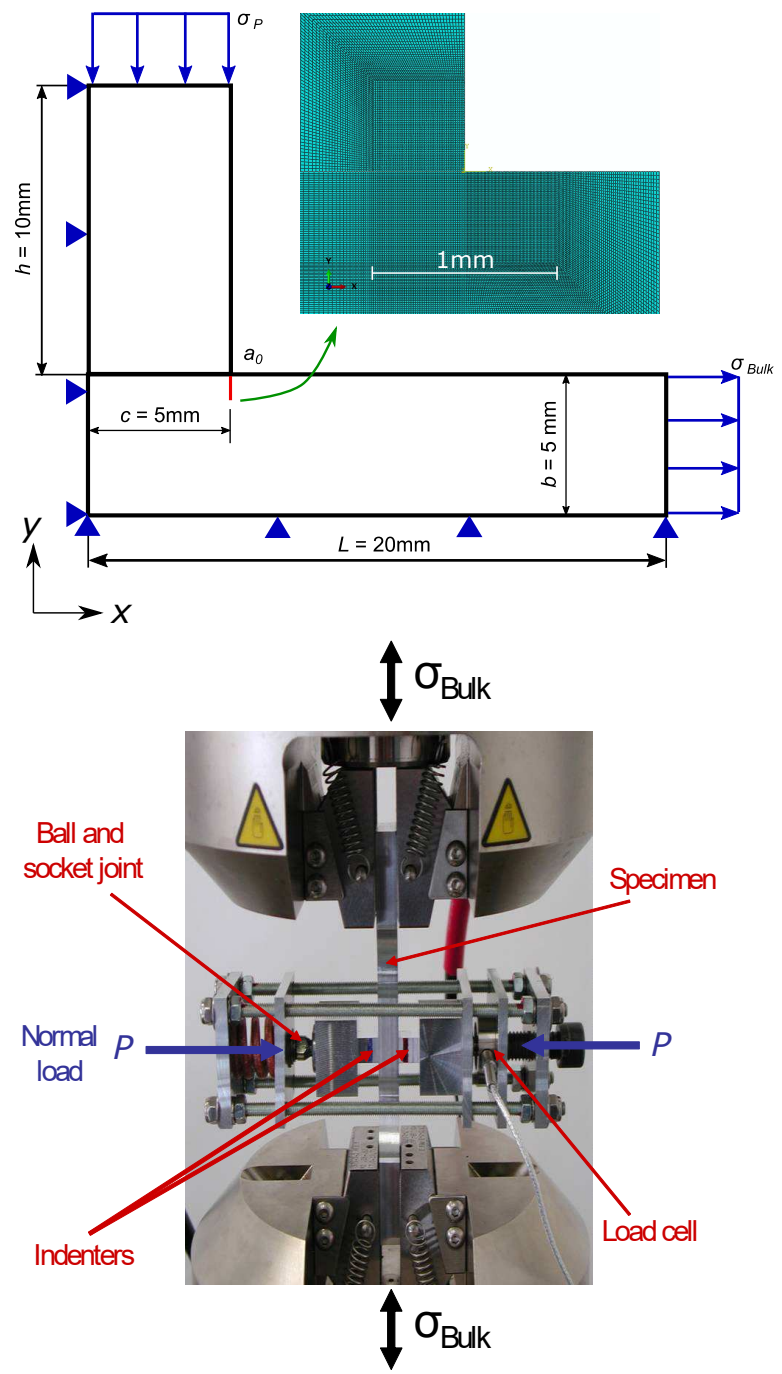

Figure 6: Top, model geometry and detail of the refined mesh at the potential crack growth zone. Bottom, complete contact testing rig, showing the contact elements.

steps have been considered in the analysis. Due to the non-linearity of the contact problem, loads were applied in sufficiently small time increments. At time $t=2.00$ (and also at time $t=4.00$ ) the maximum $\sigma_{\text {Bulk }}$ is being applied, which produces a clear opening of the crack. When the bulk load is decreased in the first half of step $3(2.00<t<2.50)$, mode I is reduced and 
a clear mixed mode condition appears, which has been observed through FE analyses. Note that the vertical load due to the indenter is kept constant during the cycle and mode II increases its dominance over mode I as $\sigma_{\text {Bulk }}$ is reduced. At approximately $t=2.50$ crack face contact is produced and a mode II condition is present at the crack tip. At time $t=3.00$ the bulk load is completely reversed (since the stress ratio is $R=-1$ ) and the load is transmitted through the crack faces. When the crack is closed, the end of the contact zone acts now as a strong stress raiser, as the specimen is compressed against the contact corner. Results in the following section are presented for the load step $4(3.00<t<4.00)$, when shakedown of the numerical model response is produced). It has been verified that the stress states at $t=3.50, t=4.00$ and those at $t=2.50, t=2.00$, respectively, are very similar.

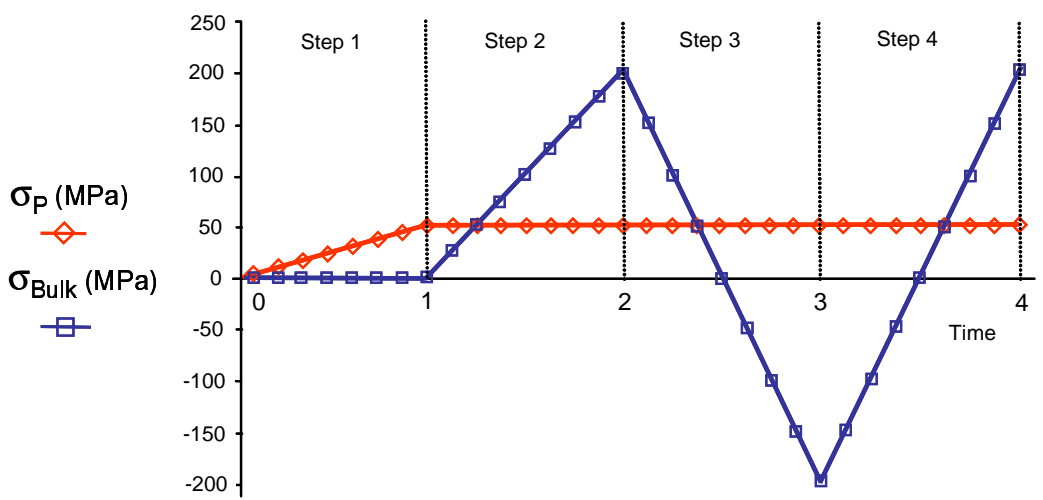

Figure 7: Loads applied to the numerical model for one of the cases studied. Evolution with time.

\section{Analysis of the loading influence}

In this section we present an initial study of the loading influence on the crack orientation. The main parameters considered are the indenter load 
$\sigma_{P}$ (defined as the normal force $P$ divided by the area of application on the indenter), the cyclic bulk load $\sigma_{\text {Bulk }}$ and the stress ratio $R$ of the cyclic bulk load. The study is performed for the first propagation angle after the initial crack shown in Fig. $6\left(a_{0}=0.3 \mathrm{~mm}\right)$, which is assumed that it is already present. The analyses were carried out with standard FE models, i.e. no XFEM is used in this section, as propagation will be considered in the next Section 5. The stress solution is then postprocessed to estimate the first propagation angle after application of the $\min (\Delta \tau)$ criterion.

The variation of the loading parameters is listed in Table 1. The geometrical model with an initial crack of length $a_{0}=0.3 \mathrm{~mm}$ and initial orientation of $\theta=90^{\circ}$ has been analyzed under 13 different cases. The cases consider different combinations of normal load $P$ applied on the indenter, the variable bulk load on the specimen $\sigma_{\text {Bulk }}$ and the stress ratio $R$. The material stiffness is $72 \mathrm{GPa}$ in all cases, both for the indenter and specimen. The last column indicates the predicted angle using the $\min (\Delta \tau)$ criterion. Contrary to what initially expected, the first fact that draws attention is that there is no practical variation of the predicted angle, since all cases lead to an orientation angle of $78^{\circ}-79^{\circ}$. Even for the cases with negligible contacting normal load, $P=10^{-6}$, the prediction leads to angles pointing inwards. The influence of the wide ranges tested for $\sigma_{\text {Bulk }}$ and $R$ is also negligible. This is in full agreement with the experimental evidence collected by the authors $[10,15]$, summarized in Fig. 2, with growing directions about $79^{\circ}$.

Fig. 8 shows the variation of $\Delta \tau$ versus the prospective crack orientation angle $\theta$ for the last step of the loading cycle. This enables the application of the minimum shear stress range criterion. Fig. 8, left, shows the results 
Table 1: Predicted orientation angles for different load cases, generated by variation of $\sigma_{P}, \sigma_{\mathrm{Bulk}}$ and $R$.

\begin{tabular}{ccccc}
\hline Case & $\sigma_{P}(\mathrm{MPa})$ & $\sigma_{\mathrm{Bulk}, \max }(\mathrm{MPa})$ & $R$ & $\theta\left(^{\circ}\right)$ \\
\hline 1 & $10^{-6}$ & 200 & -1 & 79 \\
2 & $10^{-6}$ & 200 & 0 & 79 \\
3 & 50 & 200 & -1 & 79 \\
4 & 50 & 200 & 0 & 79 \\
5 & 100 & 200 & -1 & 78 \\
6 & 100 & 200 & 0 & 79 \\
7 & 200 & 200 & -1 & 78 \\
8 & 200 & 200 & 0 & 79 \\
9 & $10^{-6}$ & 200 & -0.5 & 79 \\
10 & 50 & 200 & -0.5 & 79 \\
11 & 100 & 200 & -0.5 & 79 \\
12 & 200 & 200 & -0.5 & 79 \\
13 & 200 & 10 & -1 & 79 \\
\hline
\end{tabular}
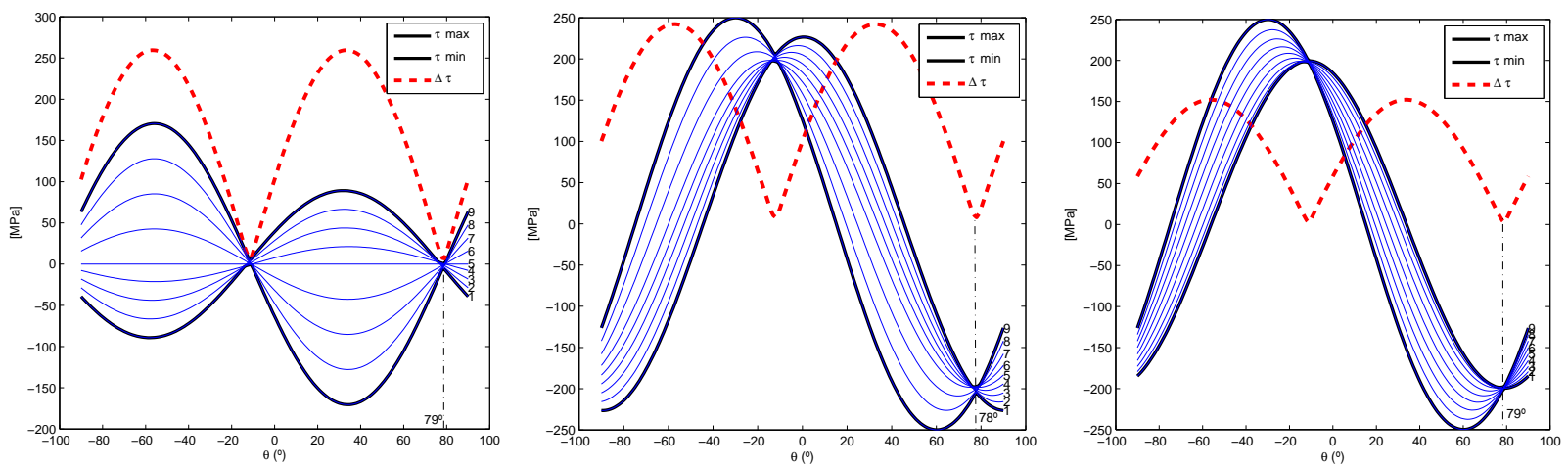

Figure 8: Application of the $\min (\Delta \tau)$ criterion for cases 1, 7 and 8 of Table 1, leading to predicted angles of $79^{\circ}, 78^{\circ}$ and $79^{\circ}$, respectively.

${ }_{269}$ for case 1 . The high proportionality of the loads is demonstrated by the 270 same location of the maxima and minima (no shifting of the curves). The 271 load proportionality is caused by the extremely low value of $\sigma_{P}$ considered 272 in this case 1 . However, even under this situation, the effect of the indenter 273 causes the deflection of the crack to $79^{\circ}$, given the ideal contact conditions 274 of the numerical model. Results for case 7 are presented in Fig. 8, centre. 275 Here the nonproportionality is evident due to the high value of $\sigma_{P}$, which is 

297 to [5] and [10].

equal to $\sigma_{\text {Bulk }}$, being the curves shifted ones with respect to each other. Due to the high value of the constant normal load, the effect of the cyclic load is less evident and the range between $\tau_{\min }$ and $\tau_{\max }$ is not so important. This range is even less for case 8 (Fig. 8, right), due to the change in $R$ from -1 to 0 . In all cases, the predicted angle is approximately $79^{\circ}$. We remark that by application of a conventional orientation criterion at the instant of maximum bulk load, such as MTS, an incorrect prediction of the crack direction is obtained (pointing outwards, see [15]). Other examples of inaccurate growth orientations using the MTS criterion under nonproportional fretting loading can be found in Figs. 6 and 7 of [5] and in [21].

\section{Study of relevant factors and prediction of propagation paths using XFEM}

In the previous section, the direction for the first crack growth increment has been estimated for different loading conditions. In this section, the extended finite element method X-FEM [16] is used in combination with the $\min (\Delta \tau)$ criterion to model propagation for successive crack growth increments. The objective is to study other factors that can be relevant for the crack orientation and compare the numerical estimations with experimental tests. In these problems, the initial crack is $a=0.05 \mathrm{~mm}$ and the crack growth increment is set as $\Delta a=0.05 \mathrm{~mm}$. The initial crack orientation is based on a critical plane analysis [29,30]. For further details, please refer 


\subsection{Crack propagation using X-FEM}

The great advantage of the X-FEM method is that the crack faces do not need to conform to the element sides of a mesh. Therefore, a single mesh can be used for virtually any arbitrary crack intersecting the mesh. This avoids remeshing and it becomes especially useful when modeling crack propagation in fatigue problems. This is accomplished through a special mathematical formulation of the FE method that includes the enrichment of the standard finite elements with additional degrees of freedom (DOFs) at the nodes. These additional DOFs are associated with the nodes of the elements that are geometrically intersected by the crack (called enriched nodes and elements, respectively). Thus, the discontinuity is included in the numerical model without modifying the discretization. The X-FEM formulation allows for a further type of enrichment for the nodes next to the crack-tip. These nodes are enriched with additional DOFs to represent the first term of the classical Williams series expansion in linear elastic fracture mechanics in terms of the displacement field. Further details can be found in [15] and references therein.

The analyses have been carried out using the X-FEM implementation developed by the authors $[17,31]$ by means of a user's subroutine linked to the commercial code ABAQUS. This implementation can take into account crack face contacts along the loading cycle, which have been proved to be essential for the correct crack prediction. Fig. 9 (left) shows paths obtained experimentally and numerically for the following parameters: $E_{\text {indenter }}=$ $E_{\text {specimen }}=72 \mathrm{GPa}(\mathrm{Al} 7075-\mathrm{T} 6), P=160 \mathrm{MPa}, \sigma_{\mathrm{Bulk}}=190 \mathrm{MPa}, R=-1$, indenter width $2 c=10 \mathrm{~mm}$ and $\mu_{\mathrm{CF}}=\mu_{\mathrm{IS}}=0.8$. A good agreement between the experimental path and the crack path predicted using X-FEM 

of the crack.
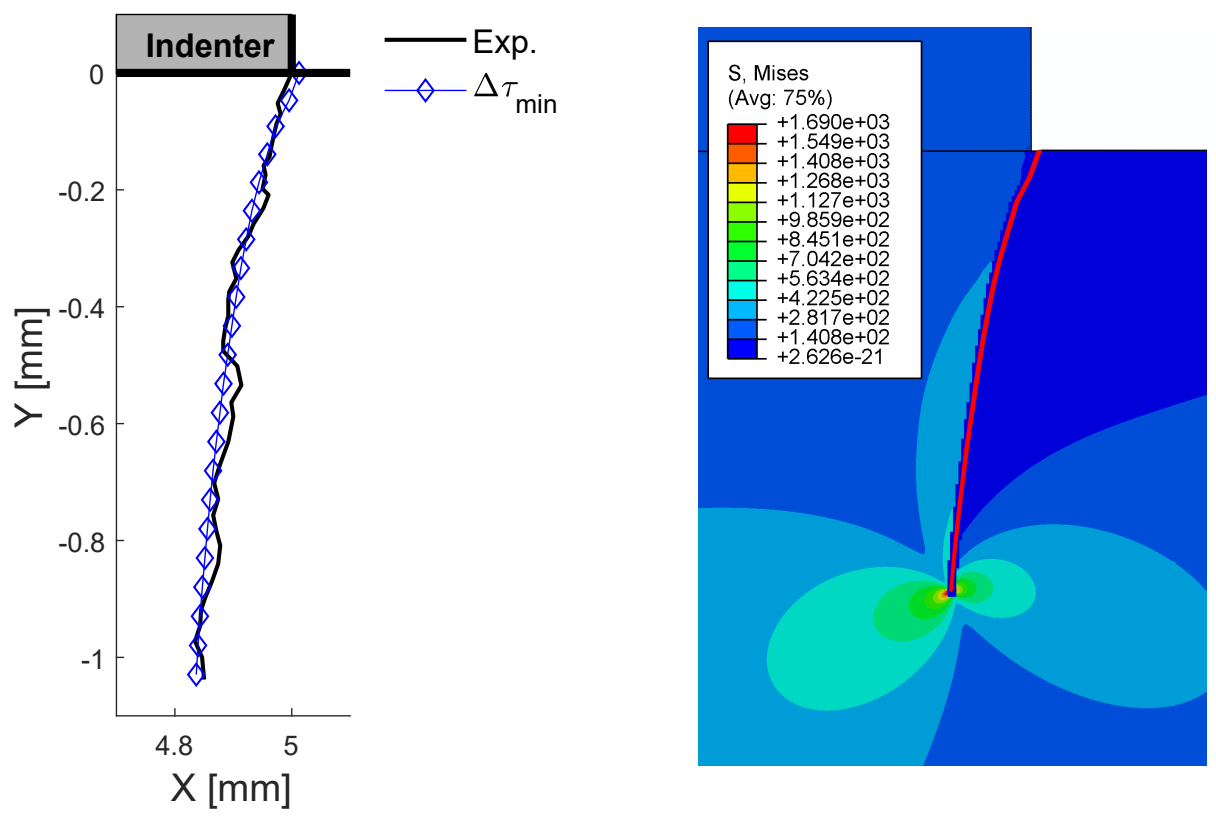

Figure 9: Left, comparison between the crack paths obtained experimentally (rightmost micrograph shown in Fig. 2) and obtained through X-FEM in combination with the $\min (\Delta \tau)$ criterion. The parameters of this problem are: $E_{\text {indenter }}=E_{\text {specimen }}=72$ $\mathrm{GPa}, P=160 \mathrm{MPa}, \sigma_{\mathrm{Bulk}, \max }=190 \mathrm{MPa}, R=-1$, indenter width $2 c=10 \mathrm{~mm}$ and $\mu_{\mathrm{CF}}=\mu_{\mathrm{IS}}=0.8$. Right, von Mises contour plot at one of the stages of the numerical simulation of crack propagation.

In this section, the influence of these factors will be analyzed: indenter normal load $P$, coefficient of friction, indenter width and indenter stiffness. In general, and unless otherwise stated, the following values are considered $E_{\text {indenter }}=72 \mathrm{GPa}\left(\mathrm{Al} 7075\right.$-T6), $E_{\text {specimen }}=72 \mathrm{GPa}, P=40 \mathrm{MPa}, \sigma_{\text {Bulk }}=$ $110 \mathrm{MPa}, R=-1$, indenter width $2 c=10 \mathrm{~mm}$ and $\mu_{\mathrm{CF}}=\mu_{\mathrm{IS}}=0.8$. This configuration is considered as the reference configuration. 


\subsection{Influence of the indenter normal load $\sigma_{P}$}

Fig. 10 (left) shows the propagation paths estimated for different values of the indenter load $\sigma_{P}$ after 14 crack growth increments. Note that a new set of loads is considered in this and following subsections because of the limitations of the testing rig shown in Fig. 6.

The results confirm the analysis given in Section 4 because it is verified that the amount of indenter load does not significantly affect the crack orientation. This is in line with the experimental evidence of Fig. 2. This unexpected behaviour was one of the motivations of this work. Although the crack path tends to grow inwards, normal loads higher than $\sigma_{P}=80$ $\mathrm{MPa}$ do not further affect the crack orientation and converge to very similar crack paths.
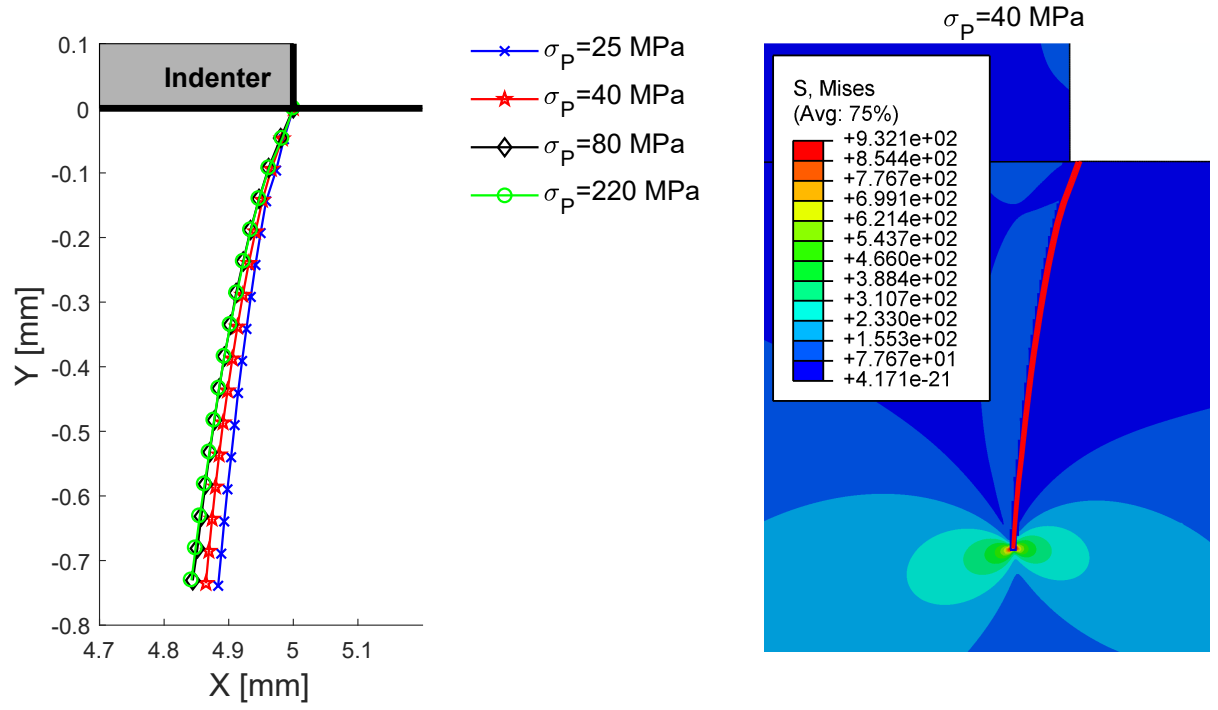

Figure 10: Left, influence of the indenter load $\sigma_{P}$. Crack propagation after 14 increments using X-FEM and the $\min (\Delta \tau)$ criterion. Right, von Mises contour plot and crack propagation for $\sigma_{P}=40 \mathrm{MPa}$ (reference problem). 
5.3. Influence of the friction coefficient $\mu_{C F}$ and $\mu_{I S}$

The potential influence of the friction coefficient between crack faces and between the indenter and specimen is addressed in this subsection. A friction coefficient of $\mu=0.8$ reported in [19] has been considered in the rest of analyses of this work for both contacts. However, a sensitivity analysis of these parameters was deemed necessary due to the uncertainty of their estimation.

Fig. 11 (left,top) shows that a wide range variation of $\mu_{\mathrm{CF}}$ between crack faces does not lead to any relevant modification of the crack path. However, Fig. 11 (left,bottom) displays a relevant influence of $\mu_{\mathrm{IS}}$ between indenter and specimen. The rest of parameters are the same as in Section 5.2, with $\sigma_{P}=40 \mathrm{MPa}$. Values close to $\mu_{\mathrm{IS}}=0.8$ and greater converge to very similar crack paths. On the other hand, there is no shear stress along the contacting plane for the limiting and ideal case of $\mu_{\mathrm{IS}}=0$. Therefore, the directions $0^{\circ}$ and $90^{\circ}$ are directions of $\min (\Delta \tau)$ and, accordingly, the crack grows in the $90^{\circ}$ direction.

\subsection{Influence of the indenter width $2 c$}

The standard width of the indenter used in this work and previous studies [15] is $2 c=10 \mathrm{~mm}$, which implies a relative ratio to specimen height $2 b$ of 1.0, see Fig. 6. Different analyses were carried out changing the indenter width and keeping the rest of parameters as in Section 5.2 with $\sigma_{P}=40$ MPa. The results shown in Fig. 12 (left) reveal that there is a large influence of the indenter width on the inclination of the crack path: the larger the indenter width, the larger the inclination of the crack path up to a point at which this inclination reaches a limit. This behaviour will be explained 

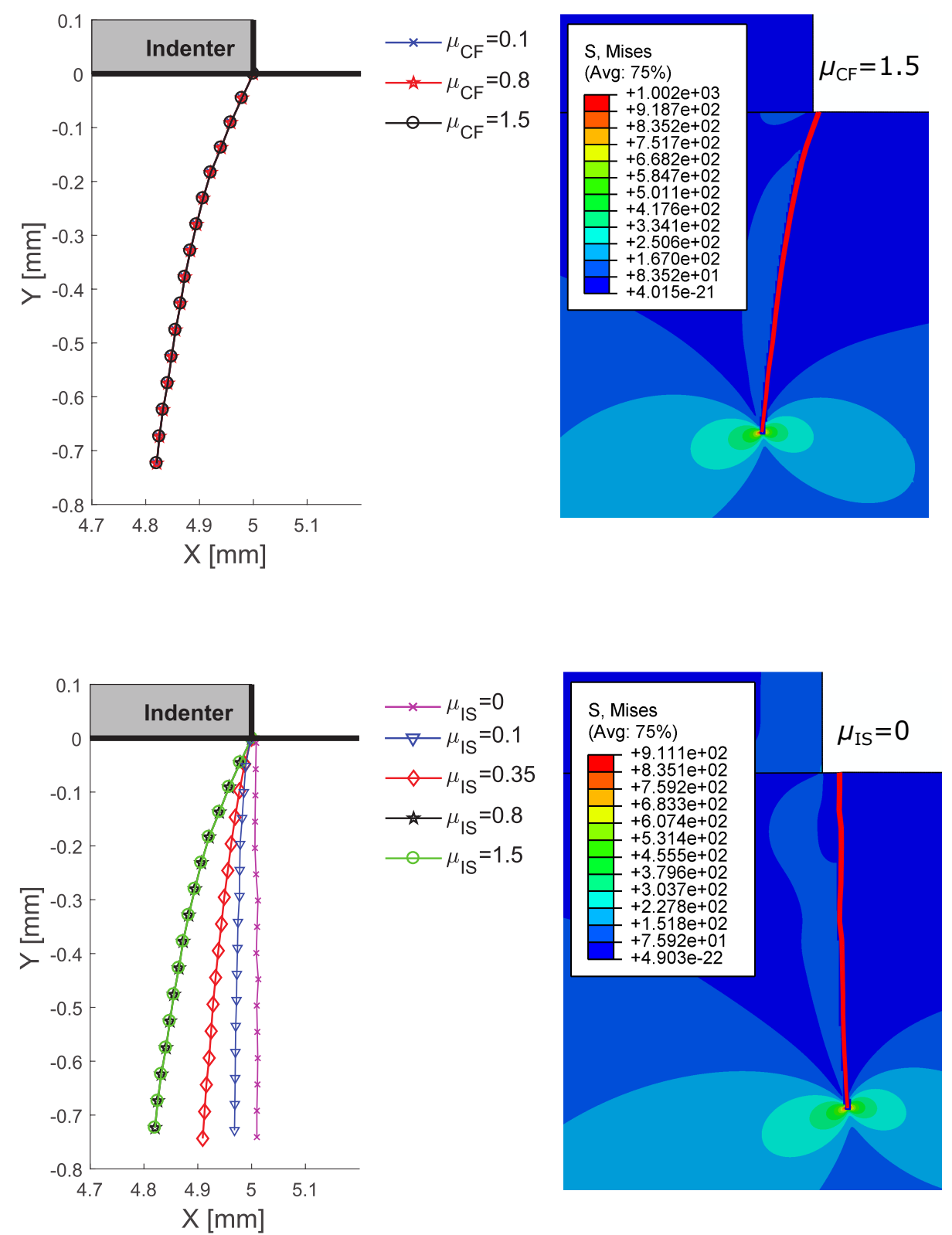

Figure 11: Top, influence of the friction coefficient $\mu_{\mathrm{CF}}$ between crack faces and $\mu_{\mathrm{IS}}$ between indenter and specimen (bottom). Crack propagation after 14 increments using $\mathrm{X}$-FEM and the $\min (\Delta \tau)$ criterion. Figures on the right show von Mises contour plots and numerical crack propagations for one case of both comparisons. 


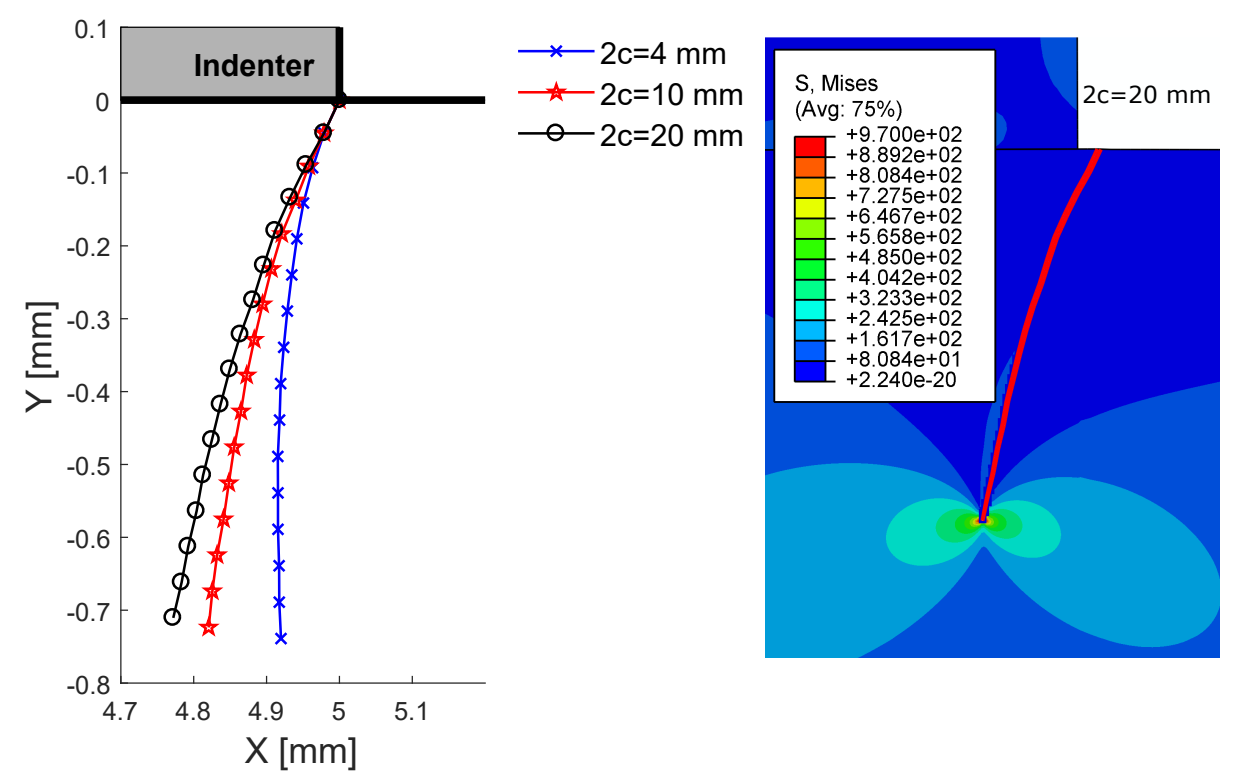

Figure 12: Left, influence of the indenter width $2 c$. Crack propagation after 10 increments using X-FEM and the $\min (\Delta \tau)$ criterion. Right, von Mises contour plot and crack propagation for the case $2 c=20 \mathrm{~mm}$.

\subsection{Influence of the indenter Young's modulus $E_{\text {indenter }}$}

Fig. 13 (left) shows the results obtained when changing the Young's modulus of the indenter, i.e. considering dissimilar materials for indenter and specimen. It can be seen that the relative stiffness of the indenter with respect to the specimen has an influence on the crack deflection inwards the contact zone. This effect, together with the influence of the indenter width, enabled us to gain insight into the mechanisms that cause the inclination of the the crack path, as explained in Section 5.7.

It can be observed that for the case of a negligible Young's modulus the predicted angle after the initial crack is close to $\theta=90^{\circ}$. The larger 

reaching a limit which corresponds to an angle of about $\theta=75^{\circ}$

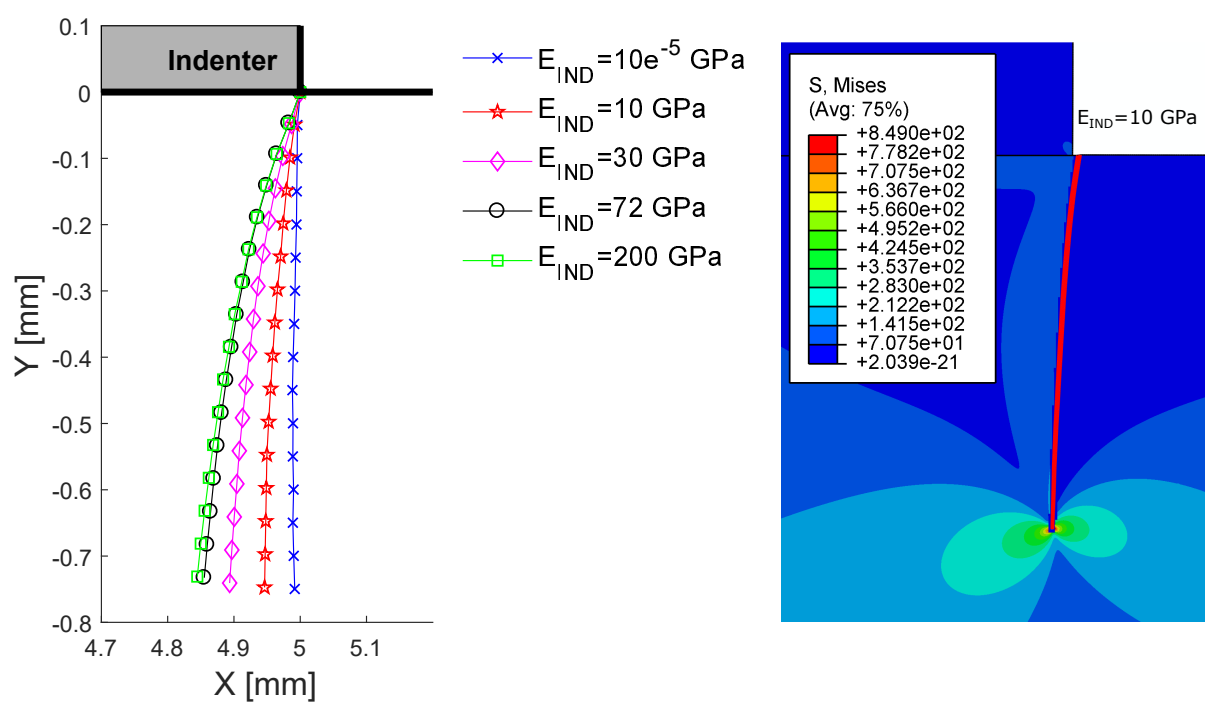

Figure 13: Left, influence of $E_{\text {indenter }}$. Crack propagation after 14 increments using XFEM and the $\min (\Delta \tau)$ criterion. $E_{\text {specimen }}=72 \cdot 10^{3} \mathrm{MPa}$ for all cases. Right, von Mises contour plot and crack propagation for the case $E_{\text {indenter }}=10 \mathrm{GPa}$.

\subsection{Experimental verification}

Figs. 14 and 15 show experimental paths found for tests with different indenter widths and different indenter Young's moduli, respectively. The loads are defined in Section 5.2, with $\sigma_{P}=40 \mathrm{MPa}$. As expected, the micrographs show that the crack inclination is slightly greater for a steel indenter than for an aluminium indenter. Fig. 14 shows a greater inclination of the path, which was also observed in the previous numerical results.

\subsection{Discussion}

It has been shown that the most relevant parameters affecting the crack orientation are the indenter width, the relative value of the indenter Young's 

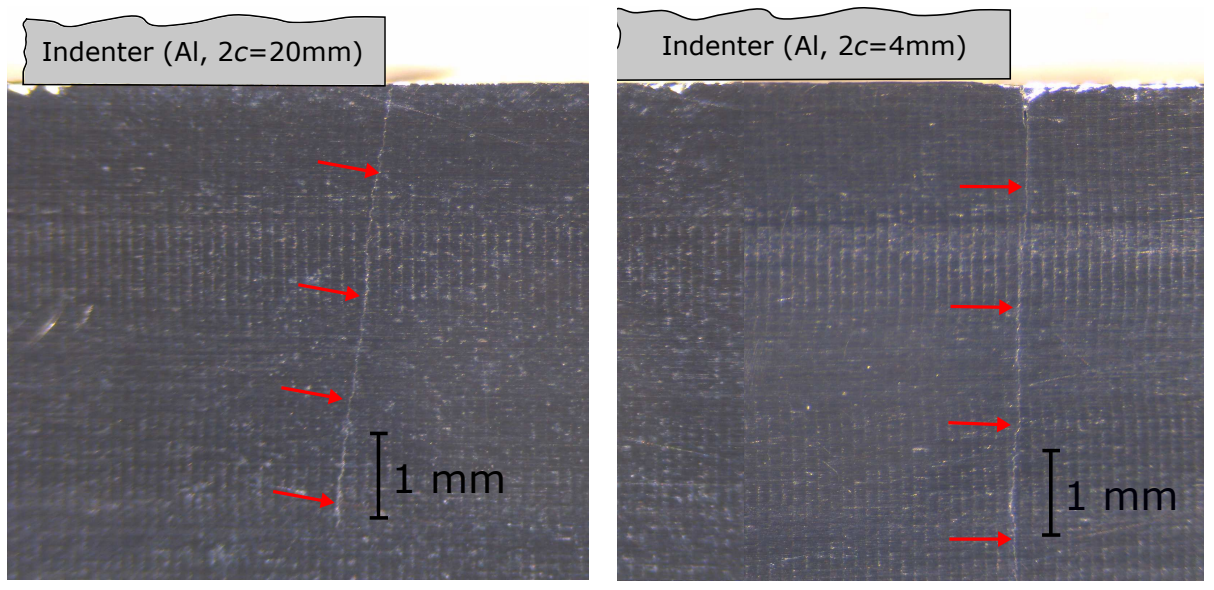

Figure 14: Influence of indenter width. Experimental paths found for two tests with aluminium indenters, width $2 c=20 \mathrm{~mm}$ (left) and width $2 c=4 \mathrm{~mm}$ (right).
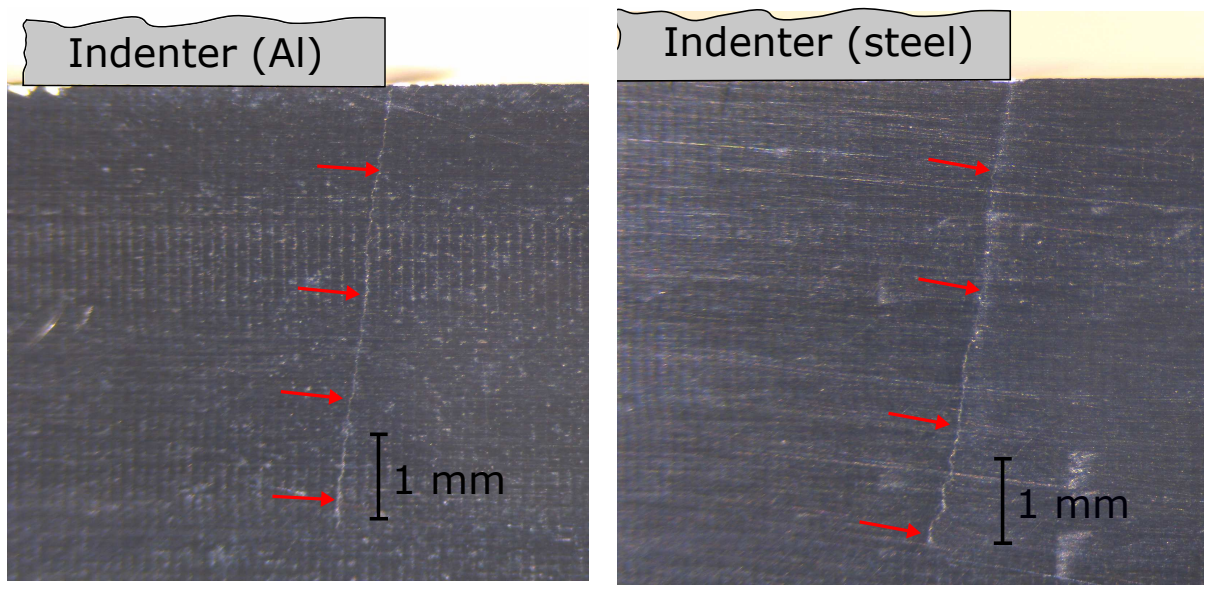

Figure 15: Influence of $E_{\text {indenter }}$. Experimental paths found for two tests with aluminium (left) and steel (right) indenters, both of width $2 c=20 \mathrm{~mm}$.

modulus with respect to the specimen and the coefficient of friction between indenter and specimen. In what follows, we provide a simple explanation for this behavior: the indenter acts as a contacting solid next to the specimen through which the force lines deviate. This is due to its stiffness and geometry, since a stiff solid tends to transfer a higher load than a compliant solid 
(assuming a parallel configuration). Similarly, a large indenter width allows for an easier force line deviation than for a small indenter width (relative to the specimen height). This can be visualized by the directions followed by the maximum principal stresses shown in Fig. 16 for one of the analysis. It can be seen that the principal directions (that can be assimilated to local force lines) tend to divert to the indenter just behind the crack. Therefore, it is expected a growth path approximately normal to the directions of the force lines in this region. The amount of deflection reaches a limit, despite a high increase of $E_{\text {indenter }}$, due to the geometric configuration of the model that does not allow for further deviation of the force lines.

For the case $\mu_{\mathrm{IS}}=0$ shown in Fig. 11, the absence of shear stresses along the contacting surface implies that the directions $0^{\circ}$ and $90^{\circ}$ are principal stress directions. Therefore, the line forces in the specimen are parallel to the contacting surface and do not divert to the indenter, leading to a crack growth in the $90^{\circ}$ direction.

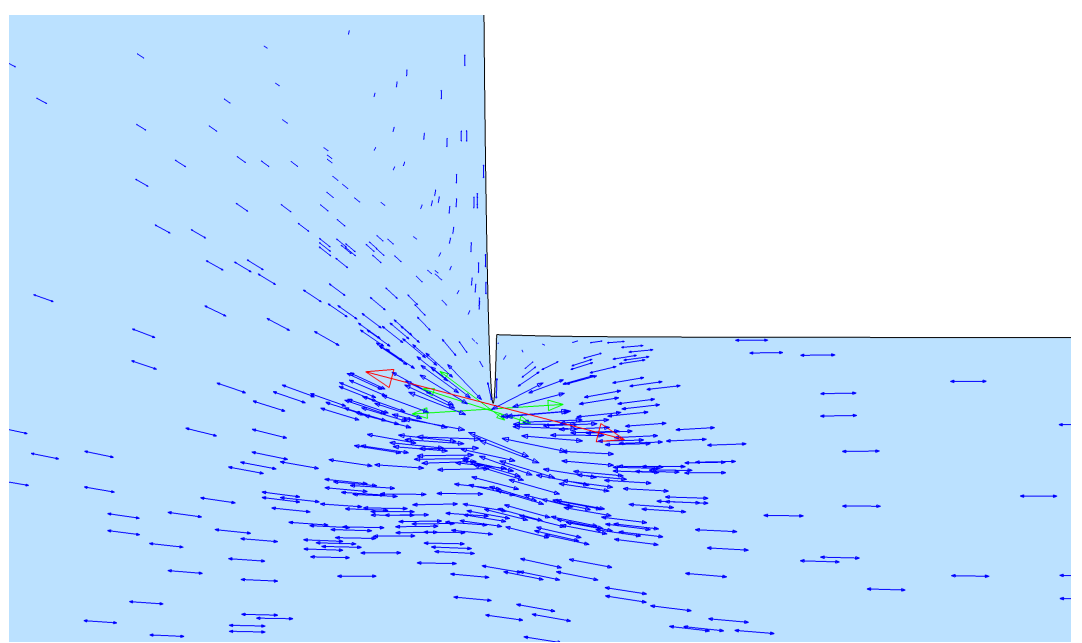

Figure 16: Deviation of the maximum principal stress directions near the contact zone. Enlarged view around the crack tip. 


\section{Conclusions}

In this work, a parametric study of the main factors affecting a fretting fatigue problem under complete contact conditions has been performed. Several parameters have been varied, such as the normal load on the indenter, the cyclic bulk load on the specimen, the stress ratio, the coefficient of friction, the indenter width and the elasticity modulus of the indenter. It has been shown that the parameters related to the loading have very little effect on the crack deflection, whereas changes of the indenter width, the indenter stiffness or the friction coefficient between indenter and specimen have a more significant effect on the crack direction.

The crack path prediction has been performed numerically using XFEM including a formulation that allows for crack face contact, which is essential to take into account the effects during the compressive part of the cycle. The approach combines XFEM with the criterion of the minimum shear stress range along the whole cycle of loading, since this type of problem is subjected to nonproportional loading, which invalidates the application of conventional orientation criteria, such as the maximum tangential stress (MTS). The numerical results are in good agreement with the experimental observations, confirming the relevant factors affecting the crack orientation. The work provides insight into the mechanisms governing crack orientation in fretting fatigue and can lead to more accurate fatigue life estimations once the crack path is predicted using the proposed procedure.

\section{Acknowledgements}

The authors gratefully acknowledge the financial support given by the Spanish Ministry of Economy and Competitiveness and the FEDER pro27 
gram through the project DPI2017-89197-C2-2-R. The support of the Generalitat Valenciana, Programme PROMETEO 2016/007, is also acknowledged. The authors thank the collaboration of Mr. Francisco Gelardo Rodríguez.

\section{References}

[1] Hills DA, Nowell D. Mechanics of Fretting Fatigue, Solid Mechanics and its Applications. 1st ed. Dordrecht, Netherlands: Kluwer Academic Publishers; 1994.

[2] Vincent L, Berthier Y, Godet M. Testing methods in fretting fatigue: a critical appraisal, in: Attia MH and Waterhouse RB (Eds.), Standardization of Fretting Fatigue Test Methods and Equipment, ASTM STP 1159, West Conshohocken, PA, USA 1992, pp. 33-48.

[3] Giannakopoulos AE, Lindley TC, Suresh S, Chenut C. Similarities of stress concentrations in contact at round punches and fatigue in notches: implications to fretting fatigue crack initiation. Fatigue Fract Engng Mater Struct 2000;23:561-571.

[4] Ciavarella M. A crack-like notch analogue for a safe-life fretting fatigue design methodology. Fatigue Fract Engng Mater Struct 2003;26:1159-1170.

[5] Giner E, Sukumar N, Denia FD, Fuenmayor FJ. Extended finite element method for fretting fatigue crack propagation. Int J Solids Struct 2008;45:5676-5687.

[6] Giner E, Tur M, Vercher A, Fuenmayor FJ. Numerical modelling of crack-contact interaction in 2D incomplete fretting contacts using X-FEM. Tribol Int 2009;42:12691275 .

[7] Giner E, Navarro C, Sabsabi M, Tur M, Domínguez J, Fuenmayor FJ. Fretting fatigue life prediction using the extended finite element method. Int J Mech Sci 2011;53:217-225.

[8] Martínez JC, Vanegas-Useche LV, Wahab MA. Numerical prediction of fretting fatigue crack trajectory in a railway axle using XFEM. Int J Fatigue 2017;100:3249 .

[9] Pereira K, Wahab MA. Fretting fatigue crack propagation lifetime prediction in 
cylindrical contact using an extended MTS criterion for non-proportional loading. Tribol Int 2017;115:525-534.

[10] Sabsabi M, Giner E, Fuenmayor FJ. Experimental fatigue testing of a fretting complete contact and numerical life correlation using X-FEM. Int J Fatigue 2011;33:811822.

[11] Sunde SL, Berto F, Haugen B. Predicting fretting fatigue in engineering design. Int J Fatigue 2018;117:314-326.

[12] Navarro C, García M, Domínguez J. A procedure for estimating the total life in fretting fatigue. Fatigue Fract Engng Mater Struct 2003;26:459-468.

[13] Pereira K, Bhatti M, Wahab MA. Prediction of fretting fatigue crack initiation location and direction using cohesive zone model. Tribol Int 2018;127:245-254.

[14] Araújo JA, Almeida GMJ, Ferreira JLA, da Silva CRM, Castro FC. Early cracking orientation under high stress gradients: The fretting case. Int J Fatigue 2017:100:611-618.

[15] Giner E, Sabsabi M, Ródenas JJ, Fuenmayor FJ. Direction of crack propagation in a complete contact fretting fatigue problem. Int J Fatigue 2014;58:172-180.

[16] Moës N, Dolbow J, Belytschko T. A finite element method for crack growth without remeshing. Int J Numer Methods Engng 1999;46:131-150.

[17] Giner E, Sukumar N, Tarancón JE, Fuenmayor FJ. An Abaqus implementation of the extended finite element method. Engng Fract Mech 2009;76:347-368.

[18] Hattori T, Nakamura M, Watanabe T. Simulation of fretting fatigue life by using stress-singularity parameters and fracture mechanics. Tribol Int 2003;36:87-97.

[19] Mutoh Y, Xu JQ, Kondoh K. Observations and analysis of fretting fatigue crack initiation and propagation, in: Kinyon SE, Hoeppner DW, Mutoh Y (Eds.), Fretting fatigue: advances in basic understanding and applications, ASTM STP 1425, West Conshohocken, PA, USA 2003, pp. 61-75.

[20] Erdogan F, Sih GC. On the crack extension path in plates under loading and transverse shear. J Basic Engng 1963;85:519-527.

[21] Fadag HA, Mall S, Jain VK. A finite element analysis of fretting fatigue crack growth behaviour in Ti-6Al-4V. Engng Fract Mech 2008;75:1384-1399.

[22] Goldstein RV, Salganik RL. Brittle fracture of solids with arbitrary cracks. Int J 
Fract 1974;10:507-523.

[23] Cotterell B, Rice JR. Slightly curved or kinked cracks. Int J Fract 1980;16:155-169.

[24] Nuismer RJ. An energy release rate criterion for mixed mode fracture. Int J Fract 1975;11:245-250.

[25] Sumi Y. Mathematical and computational analyses of cracking formation. Tokyo: Springer; 2014.

[26] Sumi Y. Fracture morphology and its evolution. A review on crack path stability and brittle fracture along butt-weld, in: Carpinteri A, Pook LP, Susmel L, Tovo R (Eds.), Proceedings of the 5th Conference on Crack Paths, Ferrara, Italy, 2015, pp. $43-59$.

[27] Giner E, Sabsabi M, Fuenmayor FJ. Calculation of $K_{\text {II }}$ in crack face contacts using X-FEM. Application to fretting fatigue. Engng Fract Mech 2011;78(2):428-445.

[28] Ribeaucourt R, Baietto-Dubourg MC, Gravouil A. A new fatigue frictional contact crack propagation model with the coupled X-FEM/LATIN method. Comput Methods Appl Mech Engng 2007;196:3230-3247.

[29] McDiarmid DL. A shear stress based critical-plane criterion of multiaxial fatigue failure for design and life prediction. Fatigue Fract Engng Mater Struct 1994;17:14751484.

[30] Fatemi A, Socie DF. A critical plane approach to multiaxial fatigue damage including out-of-phase loading. Fatigue Fract Engng Mater Struct 1988;11:145-165.

[31] Giner E, Tur M, Tarancón JE, Fuenmayor FJ. Crack face contact in X-FEM using a segment-to-segment approach. Int J Numer Methods Engng 2010;82:1424-1449. 